\title{
Tasa de crecimiento poblacional del rotífero Brachionus rotundiformis (Rotifera: Brachionidae) en un quimiostato de dos cámaras
}

\author{
María I. Cabrera F. \\ Departamento de Biología, Facultad Experimental de Ciencias, Universidad del Zulia, Laboratorio de Modelos \\ Ecológicos y Tramas Tróficas, Apdo. Postal 10 552, Maracaibo, Venezuela; gaiagua@yahoo.com
}

Recibido 11-XI-2007. Corregido 08-V-2008. Aceptado 16-V-2008.

\begin{abstract}
Population growth rate of the rotifer Brachionus rotundiformis (Rotifera: Brachionidae) in two-stage chemostat. The population growth rates of Brachionus rotundiformis were estimated in two-stage chemostat cultures. Chlorella sorokiniana was supplied continuously from a steady state culture growing with constant illumination on limiting nitrate. Rotifer growth in the second stage was limited by the rate of algal supply. The algal supply rate and rotifer population growth rate were determined by the second-stage dilution rate. The maximum population growth rate in the transient state of $B$. rotundiformis $\left(1.96\right.$ day $\left.^{-1}\right)$ was observed at $2.5 \times 10^{6} \mathrm{cel} / \mathrm{ml}$ of the algae whereas in the steady state the maximum population growth rate $\left(1.09\right.$ day $\left.^{-1}\right)$ was similar to the point Hopf's bifurcation predicted by Fussmann and was observed at $1 \times 10^{6} \mathrm{cel} / \mathrm{ml}$ of the algae. In the transient state, the rotifer's growth rate increased and the duplication time decreased at higher algal concentrations, until reaching a peak where the population growth rate begins to decrease. In the steady state, the opposite was true. The growth rates observed in this work are among the highest recorded for this rotifer in continuous cultures. Rev. Biol. Trop. 56 (3): 1149-1157. Epub 2008 September 30.
\end{abstract}

Keywords: Brachionus, chemostat, population growth rate, transient state, steady state, Hopf's bifurcation, food concentration.

Los rotíferos han sido los únicos metazoos exitosamente cultivados en quimiostatos donde el alimento es suministrado continuamente a la población objeto de estudio y el crecimiento poblacional se encuentra determinado por la tasa a la cual el nutriente limitante es suministrado al envase de cultivo. Esencialmente, el quimiostato opera proporcionando a los organismos un ambiente constante donde la tasa de crecimiento específica puede ser controlada por la tasa de dilución $D$, siendo la densidad poblacional la variable dependiente. Hasta ahora, los grandes tiempos generacionales, de otros organismos del plancton como los cladóceros, han impedido su cultivo en los sistemas de cultivo continuo. Los quimiostatos para rotíferos han sido ampliamente usados en aplicaciones prácticas. Existe abundante literatura relacionada con el cultivo de rotíferos aunque solo algunas especies de Brachionus y Keratella cochlearis han sido usadas en estos sistemas debido a los excelentes atributos que poseen ambos taxones para ser cultivados (Boraas 1983, Walz 1983, Rothhaupt 1985, Walz 1993). Los modelos matemáticos basados en la cinética de Monod explícitamente definen el crecimiento de un organismo en términos del recurso alimentario limitante, con el consumo acercándose a una asíntota al incrementar la concentración del nutriente o alimento limitante (Boraas 1983, McNair et al. 1998, Fussmann et al. 2000). Dentro de los parámetros que son necesarios conocer en la mayoría de los modelos y necesario en el cultivo de organismos como Brachionus, se encuentra la tasa de crecimiento poblacional, parámetro que mide la tasa neta de cambio del tamaño poblacional y representa el balance entre adición por reproducción y pérdidas por mortalidad. Este parámetro solo o en conjunto con otros nos da información para 
poder entender las causas de los cambios en los tamaños de las poblaciones. Más aún, la tasa de crecimiento poblacional sintetiza todos los parámetros de las tablas de vida, porque combina supervivencia, fecundidad, tiempo de desarrollo y reproducción (Miracle y Serra 1989). Dentro del género Brachionus se encuentra la especie plicatilis, que según Segers (1995) y aceptado ampliamente, comprende en realidad dos especies eurihalinas, una denominada tipo S (pequeña), conocida también como Brachionus rotundiformis y otra denominada tipo L (grande) que sería Brachionus plicatilis. En particular presentan cariotipos distintos, según Rumengan et al. (1991), Brachionus tipo $\mathrm{S}$ el número de cromosomas es $(\mathrm{n}=25)$ y Brachionus tipo L es $(\mathrm{n}=22)$. El crecimiento de $B$. plicatilis y $B$. rotundiformis se ve afectado principalmente por factores abióticos como la temperatura y la salinidad (Yufera et al. 1997, Fielder et al. 2000, Gama-Flores et al. 2005) así como por la calidad y cantidad de alimento (Maruyama et al. 1997, Hoff y Snell 1999). El estudio del efecto del alimento sobre el crecimiento de los rotíferos del género Brachionus ha sido ampliamente estudiado tanto en cultivos cerrados (Sarma et al. 2001, Fernández-Araiza et al. 2005) como en abiertos (Abu Rezk et al. 1997, Fu et al. 1997) en cepas provenientes de las zonas subtropicales. La cantidad de alimento ha sido considerada el factor más significativo que determina el tamaño poblacional ya que sometido a niveles variables de alimento Brachionus plicatilis cambia sus patrones reproductivos y la duración de su vida (Yoshinaga et al. 2000). Ahora bien, mientras que el concepto de tasa de crecimiento poblacional ha sido de importancia central en el desarrollo de la teoría de la dinámica de poblaciones, pocos estudios empíricos han considerado la variación temporal de este parámetro poblacional en detalle. Con el fin de estudiar la variación temporal de la tasa de crecimiento poblacional y el efecto que sobre la misma ejerce la cantidad de alimento el objetivo de este trabajo fue estimar las tasas de crecimiento poblacionales en estado de transición y en estado estacionario de $B$. rotundiformis a diferentes densidades del alga Chlorella sorokiniana en un quimiostato de dos cámaras.

\section{MATERIALES Y MÉTODOS}

Todos los experimentos fueron realizados usando el rotífero tipo S, Brachionus rotundiformis (longitud lórica: 72,5 $\mu$ - $235 \mu$; ancho lórica $52,5 \mu-162,5 \mu, \mathrm{n}=386$ ) aislado de una laguna salina temporal en la zona costera del Lago de Maracaibo y el alga Chlorella sorokiniana, también aislada de cuerpos de agua de la zona (Moronta 2001), a $6 \%$ de salinidad, $25{ }^{\circ} \mathrm{C}$ y 2000 lux, en un quimiostato de dos cámaras (Boraas 1983) construido en el laboratorio y ubicado dentro de un gabinete ambiental que permitió controlar la temperatura y la intensidad de la luz. La cámara del alga (1), con una capacidad de $500 \mathrm{ml}$ y la cámara del rotífero (2) con una capacidad de $250 \mathrm{ml}$. Las densidades de Chlorella utilizadas fueron $12 \times 10^{6}, 2.5 \times 10^{6}, 1.5 \times 10^{6}, 1 \times 10^{6}$, $0.6 \times 10^{6}$ y $0.4 \times 10^{6}$ cél $/ \mathrm{ml}$ porque mediciones previas de la tasa de filtración de $B$. rotundifirmis permitieron establecer que alrededor de $1 \times 10^{6}$ cél $/ \mathrm{ml}$ de $C$. sorokiniana se encuentra la concentración óptima de esta alga para el rotífero. Chlorella estuvo limitada por nitrógeno $\left(\mathrm{NO}_{3}=1.47 \mathrm{mM} / \mathrm{L}\right)$ y fue cultivada en el medio de Rodríguez-López et al. (1980). El inóculo de Brachionus usado fue $300 \mathrm{ind} / \mathrm{ml}$, el cual era introducido en la cámara de 250 $\mathrm{ml}$ al inicio de cada experimento. Diariamente se realizaron conteos tanto del alga en ambas cámaras como del rotífero. Para el conteo del alga se utilizó una cámara de Neubauer y para el conteo del rotífero una cámara de SedgwickRafter. Además se cuantificó el número de huevos por hembra del rotífero y se asignó cada individuo a una de tres categorías de edad (huevo, joven, adulto) a partir de la observación previa de cohortes en cultivos cerrados. Las tasas de dilución fueron sincronizadas con las tasas de crecimiento poblacional mediante una bomba peristáltica de vías múltiples. Los experimentos tuvieron un tiempo de duración aproximadamente de dos semanas hasta que se estabilizara el crecimiento del rotífero. Las 
tasas de crecimiento poblacionales en la fase de transición fueron calculadas usando regresión lineal del $\ln$ (Tamaño poblacional) (Graham y Wilcox 2000). Las tasas de crecimiento en estado estacionario fueron estimadas a partir del cálculo de las tasas de dilución en la cámara 2 del quimiostato. El estado estacionario es la zona de la curva poblacional relativamente aplanada que se alcanza en el quimiostato después del estado inicial de transición. Para comprobar estadísticamente la estacionariedad en tales zonas se realizó un análisis de las funciones de autocorrelación de las series temporales de las curvas poblacionales, aún cuando los tamaños muestrales no fueron los recomendados para este tipo de análisis. En tal sentido y de manera complementaria se analizaron los coeficientes de variación de las mismas zonas de la curvas utilizados por algunos autores para determinar el grado de estabilidad de la fase estacionaria (Boraas 1983, Fussmann et al. 2000).

\section{RESULTADOS}

La Figura 1 muestra los resultados de la evolución temporal del alga y del rotífero de todos los experimentos realizados en el quimiostato. B. rotundiformis mostró un incremento en su densidad poblacional al incrementar la densidad de alimento. El día en el cual se alcanzó la máxima abundancia de $B$. rotundiformis varió de acuerdo a la densidad de algas, no encontrándose ningún patrón temporal fijo. El mayor pico de abundancia poblacional de B. rotundiformis $(400 \mathrm{ind} / \mathrm{ml})$ fue obtenido cuando la densidad de C. sorokiniana en la cámara 1 del quimiostato se encontraba alrededor de los $23 \times 10^{6}$ cél $/ \mathrm{ml}$ y la densidad del alga en la cámara 2 del quimiostato estaba alrededor de las 290000 cél/ml (Figs. 1a y 1h). El valor de densidad poblacional más bajo de $B$. rotundiformis $(0.5 \mathrm{ind} / \mathrm{ml})$ se obtuvo cuando la densidad de $C$. sorokiniana en la cámara 1 del quimiostato se encontraba entre 250000 a 270 000 cél $/ \mathrm{ml}$ y la densidad del alga en la cámara 2 del quimiostato estaba en 130000 a 280000 cél/ml (Figs. 1g y 1n).
Antes de que el estado estacionario sea alcanzado en el quimiostato, la población se encuentra en estado de transición, aumentando o disminuyendo, donde todas las relaciones matemáticas del estado estacionario no pueden ser usadas.

En general, una vez estabilizado el crecimiento del alga en la cámara 1 del quimiostato se observa una fase de crecimiento exponencial del rotífero, seguida por una fase estacionaria o de disminución, también exponencial, en la cámara 2 del quimiostato. El crecimiento del alga fue regulado por el nutriente limitante y la tasa de dilución, mientras que el crecimiento del rotífero fue regulado por la densidad del alga y por la tasa de dilución. A mayores densidades del alga, la fase estacionaria del crecimiento del rotífero se observó con mayor claridad (Figs. 1h, 1i, 1j y 1k). Mientras que a menores densidades del alga (Figs. 11, 1m ), se alcanza el estado estacionario pero con oscilaciones, o se extingue la población por falta de alimento (Fig. 1n).

Los valores de $\mathrm{CV}$ en el estado estacionario oscilaron entre $6 \%$ a $24 \%$. Este ámbito de variación coincide con los obtenidos en los trabajos previos de Boraas (1983) y Fussmann et al. (2000). Según este último autor CV de la tasa de dilución por debajo del $18 \%$ indica que la población se encuentra en equilibrio y por encima del $30 \%$ que la población presenta oscilaciones alrededor del mismo. El análisis de las funciones de autocorrelación de las series temporales del rotífero también mostró una aproximación al estado estacionario en todos los casos.

La tasa de crecimiento poblacional por día ( $r$ ) en la fase de transición de $B$. rotundiformis incrementó con la densidad del alga hasta alcanzar un valor máximo (1,96 día $\left.{ }^{-1}\right)$ (Fig. 1i), cuando la densidad de $C$. sorokiniana en la cámara 1 del quimiostato se estabilizó alrededor de los 12 x $10^{6}$ cél $/ \mathrm{ml}$ (Fig. 1b) y el valor de densidad inicial en la cámara 2 fue de $2.5 \mathrm{x}$ $10^{6} \mathrm{cé} \mathrm{l} / \mathrm{ml}$ con un promedio de $1.23 \times 10^{6}$ cél/ $\mathrm{ml}$ (Cuadro 1).

La tasa de crecimiento poblacional por día $(r)$ en estado estacionario siguió un patrón 

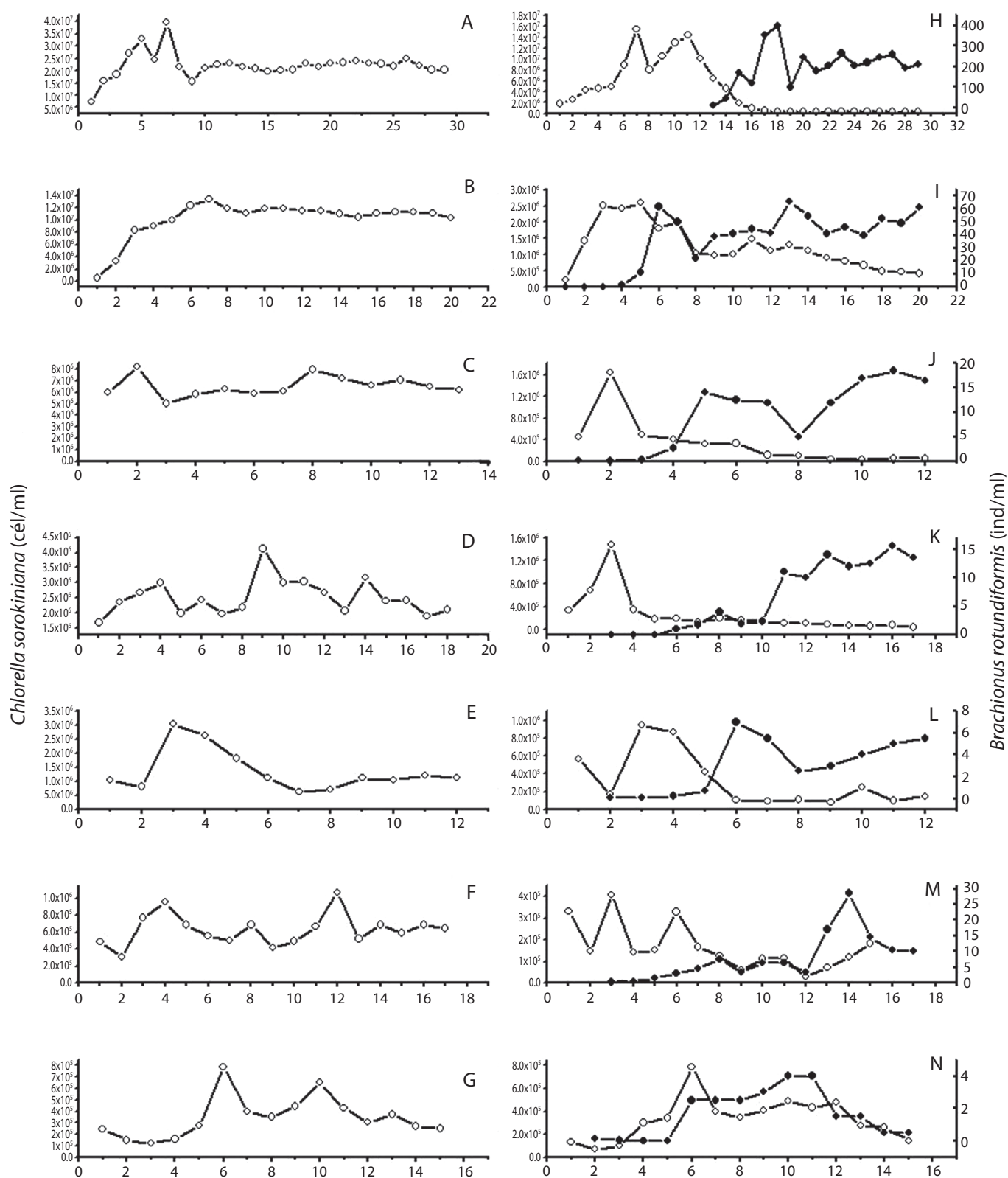

Fig. 1. a - g. Crecimiento del alga en la cámara 1 del quimiostato. $\mathrm{h}-\mathrm{n}$. Crecimiento del alga y del rotífero en la cámara 2 del quimiostato. 
CUADRO 1

Tasas de crecimiento poblacional de transición y estacionarias de Brachionus rotundiformis a diferentes concentraciones del alga en la cámara 2 del quimiostato

\begin{tabular}{|c|c|c|c|c|c|}
\hline $\begin{array}{l}\text { Densidad inicial } \\
\text { del alga (cél/ml) }\end{array}$ & $\begin{array}{l}\text { Densidad media del } \\
\text { rotífero (ind } / \mathrm{ml})\end{array}$ & $\begin{array}{c}\text { Tasa de } \\
\text { poblacior } \\
\text { transición }\end{array}$ & $\begin{array}{l}\text { ecimiento } \\
\text { (r) } \text { dia }^{-1} \\
\text { estacionaria }\end{array}$ & $\begin{array}{c}\text { Tiempo de } \\
\text { duplicación en } \\
\text { transición (horas) }\end{array}$ & $\begin{array}{c}\text { Tiempo de duplicación } \\
\text { en estado estacionario } \\
\text { (horas) }\end{array}$ \\
\hline $12 \times 10^{6}$ & 202.2 & 0.51 & 0.89 & 32.62 & 18.69 \\
\hline $2.5 \times 10^{6}$ & 35.8 & 1.96 & 1.00 & 8.49 & 16.64 \\
\hline $1.5 \times 10^{6}$ & 9.22 & 1.22 & 1.02 & 13.64 & 16.31 \\
\hline $1.5 \times 10^{6}$ & 6.67 & 0.39 & 1.01 & 42.66 & 16.47 \\
\hline $1 \times 10^{6}$ & 3.05 & 0.70 & $1.09^{*}$ & 23.77 & 15.26 \\
\hline $0.4-0.6 \times 10^{6}$ & 1.62 & 0.65 & - & 25.59 & - \\
\hline $0.4 \times 10^{6}$ & 7.85 & 0.37 & 1.03 & 44.96 & 16.15 \\
\hline
\end{tabular}

inverso al descrito anteriormente, aumentando con la disminución de la densidad del alga hasta alcanzar la tasa de crecimiento máxima, $\mu_{\max },\left(1.09\right.$ día $\left.^{-1}\right)$ (Cuadro 1).

El ámbito de valores de $r$ para B. rotundiformis en este estudio varió entre 0.37 día $^{-1}$ a 1.96 día $^{-1}$ en transición y de 0.89 día $^{-1}$ a 1.09 día $^{-1}$ en estado estacionario. El tiempo de duplicación tiende a mantenerse constante alrededor de las $16 \mathrm{hr}$ cuando se alcanza el estado estacionario mientras que durante la fase de transición es muy variable, entre 8 a $44 \mathrm{hr}$ (Cuadro 1).

De manera similar a Boraas (1983) se encontró que como funciones de la tasa de dilución $(D)$, el número total de rotíferos tiende a ser convexa (U invertida) y la densidad residual de algas es aproximadamente cóncava (forma de U) (Fig. 2).

El Cuadro 2 muestra la densidad del rotífero en estado estacionario tomando en consideración su estructura de edades y tamaño, así como la densidad algal en función de $D$. Se advierte que $D>0.0454 \mathrm{~h}^{-1}\left(=1.09 \mathrm{dí}^{-1}\right)$ es un punto crítico por encima del cual ocurre el lavado de los rotíferos. Este valor es similar al punto de bifurcación de Hopf predicho teóricamente por el modelo matemático de Fussmann et al. (2000) para B. calyciflorus de agua dulce. De igual forma que Boraas (1983), variables como el número de adultos y jóvenes tienden a ser convexas cuando se relacionan con $D$ (Fig. 3).

\section{DISCUSIÓN}

Según Suchar y Chigbu (2006) existe una fuerte correlación entre las tasas de crecimiento poblacional de los rotíferos y la abundancia de alimento. La densidad algal afecta las tasas de crecimiento poblacionales al influir sobre las tasas de alimentación e ingestión así como sobre la historia de vida de los rotíferos (Yoshinaga et al. 2003).

La tasa de crecimiento poblacional de 1.96 día $^{-1}$ para B. rotundiformis, en la fase de transición, está por encima de los valores $(<0$ $\left.\mathrm{d}^{-1}-1.35 \mathrm{~d}^{-1}\right)$ reportados por otros autores en la fase de transición de cultivos cerrados (Pascual y Yufera 1983, Snell 1986, Hirayama et al. 1989, Yoshimura et al. 1997, Gama-Flores et al. 2005), así como por los valores en cultivo 


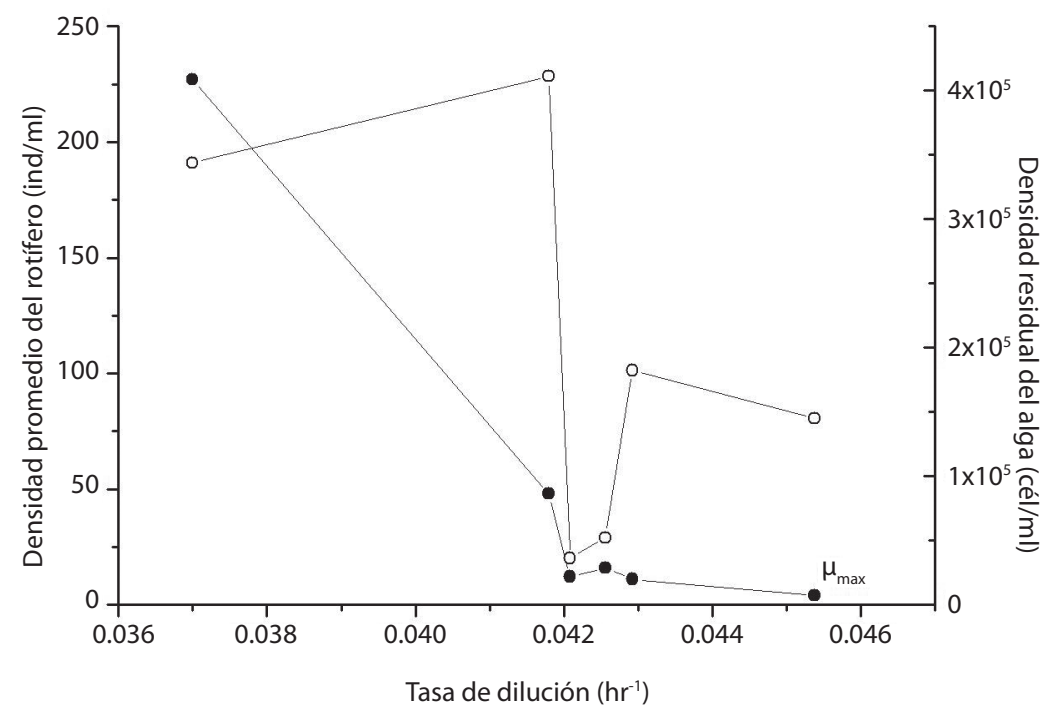

Fig. 2. Densidad promedio de B. rotundiformis y densidad residual del alga en estado estacionario en función de la tasa de dilución. Se muestra la tasa de crecimiento máxima $\left(\mu_{\max }\right)$.

CUADRO 2

Densidad del rotifero en estado estacionario (por ml), del alga (por ml.10 ${ }^{6}$ ) vs Tasa de dilución (D).

$$
\begin{array}{ccccccc}
\begin{array}{c}
D=0.0370 \\
\mathrm{~h}^{-1}(\mathrm{n}=10)
\end{array} & \begin{array}{c}
D=0.0418 \\
\mathrm{~h}^{-1}(\mathrm{n}=12)
\end{array} & \begin{array}{c}
D=0.0421 \\
\mathrm{~h}^{-1}(\mathrm{n}=7)
\end{array} & \begin{array}{c}
D=0.0426 \\
\mathrm{~h}^{-1}(\mathrm{n}=4)
\end{array} & \begin{array}{c}
D=0.0429 \\
\mathrm{~h}^{-1}(\mathrm{n}=3)
\end{array} & \begin{array}{c}
D=0.0454 \\
\mathrm{~h}^{-1}(\mathrm{n}=4)
\end{array} & \begin{array}{c}
D>0.0454 \\
\mathrm{~h}^{-1}(\mathrm{n}=0) *
\end{array} \\
\mathrm{~N}^{\mathrm{o}} & \mathrm{N}^{\mathrm{o}} & \mathrm{N}^{\mathrm{o}} & \mathrm{N}^{\mathrm{o}} & \mathrm{N}^{\mathrm{o}} & \mathrm{N}^{\mathrm{o}} & \mathrm{N}^{\mathrm{o}}
\end{array}
$$

\begin{tabular}{|c|c|c|c|c|c|c|c|}
\hline Huevos & $21 \pm 17$ & $6 \pm 4$ & $1 \pm 1$ & $1 \pm 1$ & $3 \pm 1$ & $1 \pm 1$ & $1 \pm 2$ \\
\hline Jovenes & $46 \pm 25$ & $18 \pm 6$ & $4 \pm 2$ & $5 \pm 2$ & $3 \pm 2$ & 0 & $1 \pm 1$ \\
\hline Adultos & $181 \pm 33$ & $30 \pm 13$ & $8 \pm 4$ & $11 \pm 4$ & $8 \pm 1$ & $4 \pm 1$ & $2 \pm 3$ \\
\hline Total & $223 \pm 29$ & $48 \pm 9$ & $13 \pm 2$ & $16 \pm 3$ & $12 \pm 2$ & $4 \pm 1$ & $2 \pm 1$ \\
\hline
\end{tabular}

Rotífero

\begin{tabular}{|c|c|c|c|c|c|c|c|}
\hline $\begin{array}{l}\text { Densidad } \\
\text { inicial }\end{array}$ & 12 & 2,5 & 1,5 & 1,5 & 0,4 & 1 & $0,4-0,6$ \\
\hline $\begin{array}{l}\text { Densidad } \\
\text { promedio }\end{array}$ & $\begin{aligned} & 2.2 \\
\pm & 3.96\end{aligned}$ & $\begin{array}{c}1.2 \\
\pm 0.7\end{array}$ & $\begin{array}{c}0.26 \\
\pm 0.35\end{array}$ & $\begin{array}{c}0.3 \\
\pm 0.4\end{array}$ & $\begin{array}{c}0.17 \\
\pm 0.11\end{array}$ & $\begin{array}{c}0.32 \\
\pm 0.31\end{array}$ & $\begin{array}{c}0.3 \\
\pm 0.18\end{array}$ \\
\hline Densidad final & 0.3 & 0.4 & 0.036 & 0.052 & 0.18 & 0.15 & 0.15 \\
\hline
\end{tabular}

Algas

Se muestra la Media \pm 1SD (n es el número de muestras por estado estacionario). Los valores del rotífero basados en dos réplicas por muestra. *No se alcanza el estado estacionario. 


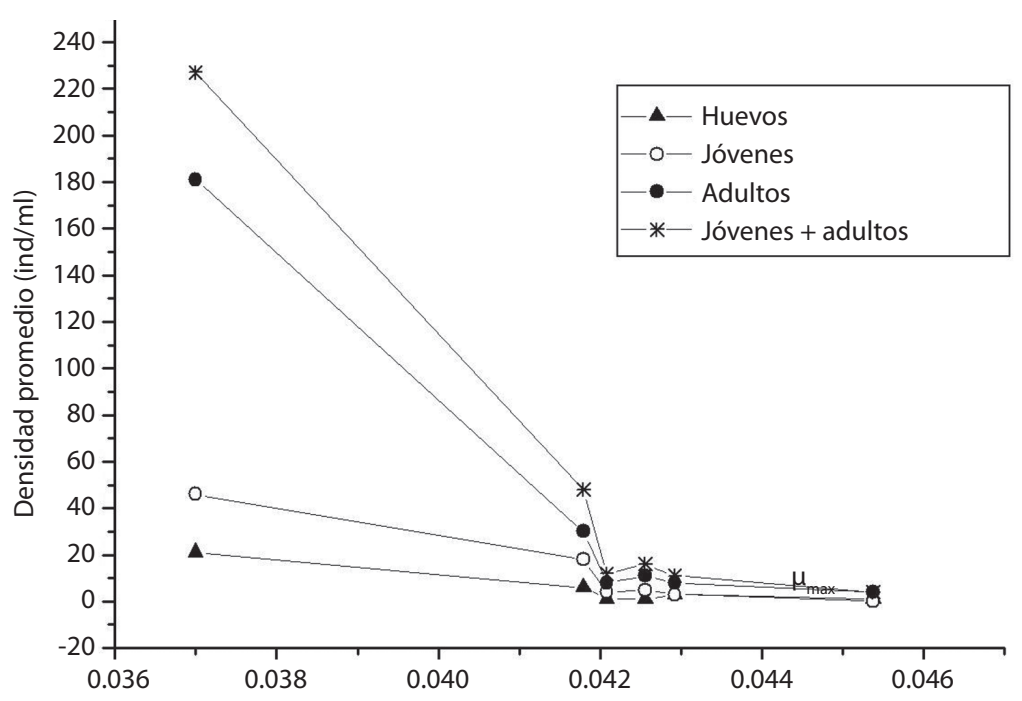

Fig. 3. Estructura de edades de B. rotundiformis en estado estacionario en función de la tasa de dilución.

cerrado obtenidos por nosotros $\left(1.2\right.$ día $\left.^{-1}\right)$. De igual manera, el valor obtenido en la fase estacionaria de 1.09 día $^{-1}\left(\mu_{\max }\right)$ en este trabajo se encuentra entre los valores mas altos reportados hasta ahora para Brachionus rotundiformis en condiciones de cultivo continuo y cercano a 1.16 día $^{-1}$, uno de los punto críticos del modelo de Fussmann et al. (2000) en donde un ciclo colapsa en un punto fijo. Los valores de tasa de crecimiento obtenidos por James y Abu Rezeq (1989) y Aoki y Hino (1996) para $B$. rotundiformis también en la fase estacionaria en sistemas de cultivo continuo $\left(0.6 \mathrm{~d}^{-1}, 1.33\right.$ $\mathrm{d}^{-1}$ respectivamente), se asemejan a los valores obtenidos en este trabajo. Estos autores sostienen que en los sistemas de flujo continuo la productividad es más alta en comparación con los sistemas convencionales cerrados. Boraas (1983) y Rothaup (1985), trabajando también en sistemas continuos pero con B. calyciflorus y $B$. rubens obtuvieron respectivamente valores de $1.18 \mathrm{~d}^{-1}$ y $0.18 \mathrm{~d}^{-1}$ en la fase estacionaria, el último marcadamente mas bajos a los valores obtenidos de B. rotundiformis. Estas diferencias reflejan las particularidades de la ecología evolutiva de las especies así como el efecto de emplear distintos alimentos y condiciones experimentales.

Los resultados comprueban la relación que existe entre la cantidad de alimento y la tasa de crecimiento de $B$. rotundiformis y que el cultivo en sistemas de flujo continuo maximiza el crecimiento siempre y cuando el alimento usado y las condiciones principalmente temperatura y salinidad se encuentren dentro de los ámbitos adecuados.

Si el valor de las elevadas tasas poblacionales de crecimiento de $B$. rotundiformis se relaciona con los datos de tasas de filtración medidos en el laboratorio (M. Cabrera, en prep.), es factible suponer que esta especie debe jugar un rol importante en las tramas tróficas de los ambientes acuáticos salinos tropicales en donde domina en conjunto con algunas especies de copépodos, sin embargo estos últimos poseen ciclos de vida mas complejos $\mathrm{y}$ tasas de filtración menores lo que convierte posiblemente a $B$. rotundiformis, en un eslabón clave en la transferencia de energía a los niveles tróficos superiores de las tramas tróficas tropicales como consecuencia de la alta tasa de renovación de sus poblaciones y de su impacto sobre las poblaciones de fitoplancton. 


\section{AGRADECIMIENTOS}

Esta investigación fue financiada por el Fondo Nacional de Investigaciones Científicas (FONACIT) a través del proyecto S1-2000000785.

\section{RESUMEN}

Se estimaron las tasas de crecimiento poblacionales de Brachionus rotundiformis en un quimiostato de dos cámaras. Suministramos Chlorella sorokiniana continuamente a partir de un cultivo en estado estacionario con iluminación constante y nitrato como nutriente limitante. El crecimiento del rotífero en la segunda cámara estuvo limitado por la tasa de suministro del alga. La tasa de suministro del alga y la tasa de crecimiento poblacional del rotífero fueron determinadas a partir de la tasa de dilución en la segunda cámara. La tasa máxima poblacional de crecimiento en estado de transición de $B$. rotundiformis (1.96 día $^{-1}$ ) se obtuvo a $2.5 \times 10^{6} \mathrm{cel} / \mathrm{ml}$ del alga, mientras que en estado estacionario la tasa máxima (1.09 $\left.\mathrm{día}^{-1}\right)$ fue similar al punto de bifurcación de Hopf predicho en Fussmann et al., (2000) y se obtuvo a $1 \times 10^{6} \mathrm{cel} / \mathrm{ml}$ del alga. En la fase de transición se observó que a mayor concentración del alga, mayor era la tasa de crecimiento del rotífero y menor su tiempo de duplicación, hasta alcanzar un pico donde comienza a decrecer. En la fase estacionaria se observó lo contrario. Los valores obtenidos se encuentran entre los más altos reportados hasta ahora para este rotífero en cultivos continuos.

Palabras Clave: Brachionus, quimiostato, tasa poblacional de crecimiento, estado de transición, estado estacionario, bifurcación de Hopf, concentración de alimento.

\section{REFERENCIAS}

Abu-Rezq, T.S., Al-Shimmari \& P. Dias. 1997. Live food production using batch culture and chemostat systems in Kuwait. Hydrobiologia 358: 173-178.

Aoki, S. \& A. Hino. 1996. Nitrogen flow in a chemostat culture of the rotifer Brachionus plicatilis. Fish. Sci. 62: $8-14$

Boraas, M.E. 1983. Population dynamics of food limited rotifers in two-stage chemostat culture. Limnol. Oceanogr. 28: 546-563.

Fernández-Araiza, M.A., S.S.S. Sarma \& S. Nandini. 2005. Combined effects of food concentration and temperature on competition among four species of Brachionus (Rotifera). Hydrobiologia 546: 519-534.
Fielder, D.S., G.J. Purser \& S.C. Battaglene. 2000. Effect of rapid changes in temperature and salinity on availability of the rotifers Brachionus rotundiformis and Brachionus plicatilis. Aquaculture 189: 85-99.

Fu, Y., A. Hada, T. Yamashita, Y. Yoshida \& A. Hino.1997. Development of a continuous culture system for stable mass production of the marine rotifer Brachionus. Hydrobiologia 358: 145-151.

Fussmann, G.F, S.P. Ellner, K.W. Shertzer \& N.G. Hairston Jr. 2000. Crossing the Hopf bifurcation in a live predator-prey system. Science 290: 1358-1360.

Gama-Flores, J.L., S.S.S. Sarma \& S. Nandini. 2005. Interaction among copper toxicity, temperature and salinity on the population dynamics of Brachionus rotundiformis (Rotifera). Hydrobiologia 546: 559568 .

Graham, L.E. \& L.W. Wilcox. 2000. Algae. Prentice Hall, Upper Saddle River, Nueva Jersey, EEUU.

Hirayama, K., I. Maruyama \& T. Maeda. 1989. Nutritional effect of freshwater Chlorella on growth of the rotifer Brachionus plicatilis. Hydrobiologia 186/ 187: 39-42.

Hoff, F. H. \& T.W. Snell. 1999. Plankton Culture Manual. Florida Aquafarms Inc. Dade City, Florida, EEUU.

James, C.M. \& T. Abu Rezeq. 1989. Intensive rotifer cultures using chemostats. Hydrobiologia 186/187: 423-430.

Maruyama, I., T. Nakao, I. Shigeno, Y. Ando \& K. Hirayama. 1997. Application of unicellular algae Chlorella vulgaris for the mass culture of marine rotifer Brachionus. Hydrobiologia 358: 133-138.

McNair, J.N., M.E. Boraas \& D.B. Seale. 1998. Sizestructure dynamics of the rotifer chemostat: a simple physiologically structured model. Hydrobiologia, 387/388: 469-476.

Miracle, M.R. \& M. Serra. 1989. Salinity and temperature influence in rotifer life history characteristics. Hydrobiologia 186/187 : 81-102.

Moronta, R.M. 2001. Caracterización bioquímica y fisiológica de la microalga autóctona Chorella sp. en condiciones autotróficas, mixotróficas y heterotróficas. Estudio de su flora microbiana asociada. Trabajo Especial de Grado, Maracaibo, Venezuela.

Pascual, E. \& M. Yufera. 1983. Crecimiento en cultivo de una cepa de Brachionus plicatilis O. F. Muller en función de la temperatura y la salinidad. Inv. Pesq. 47: 151-159. 
Rodríguez-López, M., M. Villarroya \& M. L. Muñoz-Calvo. 1980. Influence of ammonium and nitrate on protein content amino acid pattern. Storage materials and fine structure of Chlorella $8 \mathrm{H}$ recovering from N- starvation, p. 723-731. In: G. Shelef \& C.J.Soeder (eds.). Algae Biomasa. Elsevier, Amsterdam, Holanda.

Rothaup, K.O. 1985. A model approach to the population dynamics of the rotifer Brachionus rubens in twostage chemostat culture. Oecol. 65: 252-259.

Rumengan, I. F., M. A. Kayano y K. Hirayama. 1991. Karyotypes of S-type and L-type rotifers Brachionus plicatilis (Muller). J. Exp. Mar. Biol. Ecol. 154: 171-176.

Sarma, S.S.S., P. S. Larios Jurado y S. Nandini. 2001. Effect of three food types on the population growth of Brachionus calyciflorus and Brachionus patulus (Rotifera: Brachionidae). Rev. Biol. Trop. 49: 77-84.

Segers, H. 1995. Nomenclatural consequences of some recent studies on Brachionus plicatilis (Rotifera, Brachionidae). Hydrobiología. 313/314: 121-122.

Snell, T.W. 1986. Effect of temperature, salinity and food level on sexual and asexual reproduction in Brachionus plicatilis (Rotifera). Mar. Biol. 92: 157-162.

Suchar V.A. \& P. Chigbu. 2006. The effects of algae species and densities on the population growth of the marine rotifer, Colurella dicentra. J. Exp. Mar. Biol. Ecol. 337: 96-102.

Walz, N. 1983. Continuous culture of the pelagic rotifers Keratella cochlearis and Brachionus angularis. Arch. Hydrobiol. 98: 70-92.

Walz, N. 1993. Model simulations of continuous rotifer cultures. Hydrobiologia 255/256: 165-170.

Yoshimura K., K. Usuki, T. Yoshimatsu, Ch. Kitajima \& A. Hagiwara. 1997. Recent development of a high density mass culture system for the rotifer Brachionus rotundiformis Tschugunoff. Hydrobiologia 358: 139144.

Yoshinaga, T., A. Hagiwara, K. Tsukamoto. 2003. Life history response and age-specific tolerance to starvation in Brachionus plicatilis O.F. Muller (Rotifera). J. Exp. Mar. Biol. Ecol. 287: 261-271.

Yúfera, M. \& N. Navarro. 1995. Population growth dynamics of the rotifer Brachionus plicatilis cultured in non-limiting food condition. Hydrobiologia 313/314: 399-405.

Yúfera M., G. Parra \& E. Pascual. 1997. Energy content of rotifers (Brachionus plicatilis and Brachionus rotundiformis) in relation to temperature. Hydrobiologia 358: 83-87. 
